\title{
A brief introduction to Quillen conjecture
}

\author{
Bui Anh Tuan ${ }^{*}$, Nguyen Anh Thi
}

Faculty of Mathematics and Computer Science University of Science, VNU-HCMC

\section{Correspondence}

Bui Anh Tuan, Faculty of Mathematics and Computer Science University of Science, VNU-HCMC

Email: batuan@hcmus.edu.vn

\section{History}

- Received: 2018-12-05

- Accepted: 2019-03-31

- Published: 2019-06-14

DOI :

https://doi.org/10.32508/stdj.v22i2.1229

\section{Check for updates}

\section{Copyright}

(C) VNU-HCM Press. This is an openaccess article distributed under the terms of the Creative Commons Attribution 4.0 International license.

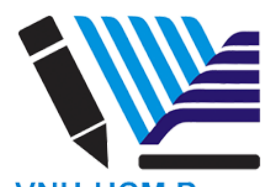

\begin{abstract}
Introduction: In 1971, Quillen stated a conjecture that on cohomology of arithmetic groups, a certain module structure over the Chern classes of the containing general linear group is free. Over time, many efforts has been dedicated into this conjecture. Some verified its correctness, some disproved it. So, the original Quillens conjecture is not correct. However, this conjecture still has great impacts on the field cohomology of group, especially on cohomology of arithmetic groups. This paper is meant to give a brief survey on Quillen conjecture and finally present a recent result that this conjecture has been verified by the authors. Method: In this work, we investigate the key reasons that makes Quillen conjecture fails. We review two of the reasons: 1) the injectivity of the restriction map; 2) the non-free of the image of the Quillen homomorphism. Those two reasons play important roles in the study of the correctness of Quillen conjecture. Results: In section 4, we present the cohomology of ring $H^{*}\left(S L_{2}\left(\mathbb{Z}[\sqrt{-2}]\left[\frac{1}{2}\right]\right) ; \mathbb{F}_{2}\right)$ which is isomorphic to the free module $\mathbb{F}_{2}\left[e_{4}\right]\left(x_{2}, x_{3}, y_{3}, z_{3}, s_{3}, x_{4}, s_{4}, s_{5}, s_{6}\right)$ over $\mathbb{F}_{2}\left[e_{4}\right]$. This confirms the Quillen conjecture. Conclusion: The scope of the conjecture is not correct in Quillens original statement. It has been disproved in many examples and also been proved in many cases. Then determining the conjectures correct range of validity still in need. The result in section 4 is one of the confirmation of the validity of the conjecture.

Key words: Quillen conjecture, Cohomology of group, Arithmetic groups, S-arithmetic groups, Cohomology ring
\end{abstract}

\section{PRELIMINARIES}

In this section, we will review some concepts related to Quillen conjecture and then give a full and details of the conjecture. The key objects in the conjecture are arithmetic and $S$-arithmetic groups whose concepts arose when studying modular form and some other classical topics in number theory. However, the definition of arithmetic groups is very technical. We can, roughly, think of arithmetic groups as the intersection of $S L(n, \mathbb{Z})$ (or $G L(\mathrm{n}, \mathbb{Z})$ ) with some semi-simple Lie subgroup $G$ of $S L(n, \mathbb{R})$ (or $G L(n, \mathbb{Z})$ ). Full definition can be seen in ${ }^{1}$ as follows:

Definition 1 Let $G$ be a semi-simple subgroup of $S L(n, \mathbb{R})$. Group $\Gamma$ is said to be an arithmetic subgroup of $G$ if and only if $\Gamma$ is a lattice in $G$ and there exist a closed, connected, semisimple subgroup $G^{\prime}$ of some $S L(n, \mathbb{R})$, such that $G^{\prime}$ is defined over $\mathbb{Q}$,

- a closed, connected, semisimple subgroup $G^{\prime}$ of some $S L(n, \mathbb{R})$, such that $G^{\prime}$ is defined over $\mathbb{Q}$,

- compact normal subgroups $K$ and $K^{\prime}$ of $G^{\circ}$ and $G^{\prime}$, respectively, and

- an isomorphism $\psi: G^{\circ} / K \rightarrow G^{\prime} / K^{\prime}$, such that $\psi(\Gamma)$ is commensurable to $\overline{G_{\mathbb{Z}}^{\prime}}$, where $\bar{\Gamma}$ and
$\overline{G_{\mathbb{Z}}^{\prime}}$ are images of $\Gamma \cap G^{\circ}$ and $G_{\mathbb{Z}}^{\prime}$ in $G^{\circ} / K$ and $G^{\prime} / K^{\prime}$, respectively.

Reader can find definition of defined over, commensurable and other related concepts in ${ }^{2}$. Some common arithmetic groups are $S L_{n}(\mathbb{Z}), P S L_{n}(\mathbb{Z}), P G L_{n}(\mathbb{Z})$ modular groups, Siegel groups, etc.

In order to generalize the concept of arithmetic groups, one natural method is replacing the ring $\mathbb{Z}$ of integers by a bigger ring. There are two ways that lead to two different branches. One is considering the ring of integers of some number fields, this leads to the concepts of Hilbert modular and Bianchi groups. Another is replacing $\mathbb{Z}$ by the ring of integers with some primes inverted $\mathbb{Z}\left[\frac{1}{p_{1}}, \frac{1}{p_{2}}, \cdots, \frac{1}{p_{n}}\right]$. The fusion of these two ideas leads us to the definitions of $S$ integers and $S$-arithmetic groups.

Recall that given an algebraic number field $K$, an absolute value on $K$ is a map $|.|_{v}: K \rightarrow \mathbb{R}_{\geq 0}$ satisfies

- $|\alpha|_{v}=0$ if and only if $\alpha=0$,

- $|\alpha \beta|_{v}=|\alpha|_{v}|\beta|_{v}$ for all $\alpha, \beta \in K$,

- there exists $a>0$ such that $|\alpha+\beta|_{v}^{a} \leq|\alpha|_{v}^{a}+$ $|\beta|_{v}^{a}$. 
One can prove that two absolute values are equivalent if there exists $c>0$ such that $|\alpha|_{1}=|\alpha|_{2}^{c}$. An equivalent class of non-trivial absolute values is called a place of $K$.

Definition 2 Let $K$ be a number field. Given a finite set $S$ of places of $K$ that containing all archimedean places. We define the ring of $S$-integers to be the set

$$
\mathscr{O}_{K, S}=\left\{a \in K \|\left. a\right|_{v} \leq 1 \text { for all } v \notin S\right\}
$$

Definition 3 Let $G$ be an algebraic group defined over $K$. Then an $S$-arithmetic subgroup $\Gamma$ of $G(K)$ is a $s u b$ group commensurable with $G(\mathscr{O} k, S)$.

Let us consider one example as follows:

Example 1 In this example, we will calculate the possibilities of $S$-integers in case $K$ is the field $\mathbb{Q}$ of rational numbers. Let $p$ be a prime, then each rational number $q \in \mathbb{Q}^{*}$ can be written uniquely as $q=p^{r} \frac{m}{n^{\prime}}$, where $(m, n)=1$ and both $m$ and $n$ are not divisible by $p$. The $p$-adic valuation $v_{p}: \mathbb{Q} \rightarrow \mathbb{Z}$ is defined by $v_{p}(q)=$ rand $v_{p}(0)=\infty$. And the $p$-adicabsolute value associated to the prime $p$ is defined $|q|_{p}=p^{-v_{p}(q)}$. Ostrowskis theorem ${ }^{3}$ says that every non-trivial absolute value on $\mathbb{Q}$ is equivalent to $|\cdot|_{p}$ for some prime $p \leq \infty$. Note that we consider the usual absolute value on $\mathbb{Q}$ as $|.|_{\infty}$, the case that $p=\infty$. Forconvenience we identify the absolute value $v_{p}$ by its related prime $p$. Thus the set of places $S$ is of the form $P \cup\{\infty\}$ where $P$ is a set of prime numbers.

Suppose that $S=\left\{\infty, p_{1}, p_{2}, \ldots, p_{n}\right\}$ then

$$
\mathscr{O}_{Q, S}=\mathbb{Z}_{S}=\mathbb{Z}\left[\frac{1}{p_{1}}, \frac{1}{p_{2}}, \cdots, \frac{1}{p_{n}}\right]
$$

And thus we have a class of $S$-arithmetic groups $S L\left(n, \mathbb{Z}\left[\frac{1}{m}\right]\right)$ where $m$ is square-free positive integer.

\section{QUILLENS CONJECTURE AND RELATED RESULTS}

In this section, we will present the details of Quillens conjecture and also give a comprehensive list of the results related to this conjecture.

Conjecture $1^{2}$ Let $\ell$ be a prime number. Let $K$ be a number field with $\zeta_{\ell} \in K$, and $S$ a finite set of places containing the infinite places and the places over $\ell$. Then the natural inclusion $\mathscr{O}_{K, S} \hookrightarrow \mathbb{C}$ makes $H^{*}\left(G L_{n}\left(\mathscr{O}_{K, S}\right) ; \mathbb{F}_{\ell}\right)$ a free module over the cohomology ring $H_{c t s}^{*}\left(G L_{n}(\mathbb{C}) ; \mathbb{F}_{\ell}\right)$.

This conjecture was re-stated as

Conjecture $2 H^{*}\left(\Gamma, \mathbb{F}_{\ell}\right)$ is a free module over the subring $\mathbb{F}_{\ell}\left[c_{1}, c_{2}, \cdots, c_{n}\right]$, where $\Gamma=G L_{n}(\mathscr{O})$ and $\mathscr{O}$ is the ring of $S$-integers in a number field and $c_{i}$ is some Chern classes.
Over the last forty years, many efforts have been put into this conjecture and many results have been published. Some of them show the positiveness of the conjecture, some disproved it. Here we give a brief list of the outstanding results.

Positive cases, in which the conjecture has been established, are:

1. $n=\ell=2, \mathscr{O}_{k, S}=\mathbb{Z}\left[\frac{1}{2}\right]$ by Mitchell ${ }^{4}$ in 1992

2. $n=3, \ell=2, \mathscr{O}_{k, S}=\mathbb{Z}\left[\frac{1}{2}\right]$ by Henn ${ }^{5}$ in 1997

3. $n=\ell=2, \mathscr{O}_{k, S}=\mathbb{Z}\left[i, \frac{1}{2}\right]$ by Weiss (supervised by Henn) in 2007

4. $n=\ell=2, \mathscr{O}_{k, S}=\mathbb{Z}\left[\sqrt{-2}, \frac{1}{2}\right]$ by Bui \& $\operatorname{Rahm}^{6}$ in 2017

Despite of the above positive cases, many mathematicians have been trying to find a counterexample. In 1998, Mitchell ${ }^{4}$ show that the restriction map

$H^{*}\left(G L_{n}\left(\mathbb{Z}\left[\frac{1}{2}\right]\right), \mathbb{F}_{2}\right) \rightarrow H^{*}\left(T_{n}\left(\mathbb{Z}\left[\frac{1}{2}\right]\right), \mathbb{F}_{2}\right)$

from $G L_{n}\left(\mathbb{Z}\left[\frac{1}{2}\right]\right)$ to the subgroup $T_{n}\left(\mathbb{Z}\left[\frac{1}{2}\right]\right)$ of diagonal matrices is isomorphic to

$$
F_{2}\left[w_{1}, w_{2}, \ldots, w_{n}\right] \otimes \Lambda\left[e_{1}, e_{2}, \ldots, e_{2 n-1}\right]
$$

Henn, Lannes and Schwartz ${ }^{7}$ show that, in the case of the Quillen's conjecture for $G L_{n}\left(\mathbb{Z}\left[\frac{1}{2}\right]\right)$ the above restriction map is injective. This shed the light on how to find counterexamples. The non-injectivity of the restriction map has been shown in some cases:

1. $n \geq 32$ and $\ell=2$ by Dwyer ${ }^{8}$

2. $n \geq 14$ and $\ell=2$ by Henn and Lannes ${ }^{9}$

3. $n \geq 27$ and $\ell=3$ by Anton ${ }^{10}$

\section{QUILLEN CONJECTURE FOR FARRELL-TATE COHOMOLOGY}

In effort of making a refinement of Quillens conjecture, Rahm and Wend, in their paper ${ }^{11}$, state that the conjecture is true for Farrel-Tate cohomology in the following cases.

Theorem $1^{11}$ Let $\ell$ be a prime number. Let $K$ be $a$ number field with $\zeta_{\ell} \in K$, and $S$ a finite set of places containing the infinite places and the places over $\ell$

1. The Quillen conjecture is true for FarrellTate cohomology of $S L_{2}\left(\mathbb{O}_{K, S}\right)$. More precisely, the natural morphism $\mathbb{F}_{\ell}\left[c_{2}\right] \cong H_{c t s}\left(S L_{2}(\mathbb{C}), \mathbb{F}_{\ell}\right) \rightarrow$ $H^{\cdot}\left(S L_{2}\left(\mathscr{O}_{K, S}\right), \mathbb{F}_{\ell}\right)$ extends to a morphism

$$
\phi: \mathbb{F}_{\ell}\left[c_{2}, c_{2}^{-1}\right] \rightarrow \widehat{H}^{*}\left(S L_{2}\left(\mathscr{O}_{K, S}\right), \mathbb{F}_{\ell}\right)
$$


which makes $\widehat{H}^{\bullet}\left(S L_{2}\left(\mathscr{O}_{K, S}\right), \mathbb{F}_{\ell}\right)$ a free $\mathbb{F}_{\ell}\left[c_{2}, c_{2}^{-1}\right]$ module.

2. The Quillen conjecture is true for $S L_{2}\left(\mathscr{O}_{K, S}\right)$ in cohomological degrees above the virtual cohomological dimension.

They also refine the conjecture to

Conjecture $3^{11}$ Let $K$ be a number field. Fix a prime $\ell$ such that $\zeta_{\ell} \in K$, and an integer $n<\ell$. Assume that $S$ is a set of places containing the infinite places and the places lying over $\ell$. If each cohomology class of $G L_{n}\left(\mathscr{O}_{K, S}\right)$ is detected on some finite subgroup, then $H_{c t s}^{\bullet}\left(G L_{n}(\mathbb{C}), \mathbb{F}_{\ell}\right)$ is a free module over the image of the restriction map $H_{c t s}^{\bullet}\left(G L_{n}(\mathbb{C}), \mathbb{F}_{\ell}\right) \rightarrow$ $H^{\bullet}\left(G L_{n}(\mathbb{C}), \mathbb{F}_{\ell}\right)$.

By the above theorem, Rahm and Wendt have made the following observations for $S L_{2}$ over rings of $S$ integers at odd prime $\ell$ :

1. The original Quillen conjecture holds for group cohomology $H^{*}\left(S L_{2}\left(\mathscr{O}_{k, S}\right) ; \mathbb{F}_{\ell}\right)$ above the virtual cohomology dimension.

2. The refined Quillen conjecture holds for FarrellTate cohomology $\widehat{H}^{*}\left(S L_{2}\left(\mathscr{O}_{k, S}\right) ; \mathbb{F}_{\ell}\right)$

\section{RECENT RESULTS}

In this section, we will introduce some results related to Quillen conjecture that the first author and his collaborator had published.

Theorem $2^{6}$

The cohomology ring $H^{*}\left(S L_{2}\left(\mathbb{Z}[\sqrt{-2}]\left[\frac{1}{2}\right]\right) ; \mathbb{F}_{2}\right)$ is isomorphic to the free module

$$
\mathbb{F}_{2}\left[e_{4}\right]\left(x_{2}, x_{3}, y_{3}, z_{3}, s_{3}, x_{4}, s_{4}, s_{5}, s_{6}\right)
$$

over $\mathbb{F}_{2}\left[e_{4}\right]$ (the image of $H_{c t s}^{*}\left(S L_{2}(\mathbb{C}), \mathbb{F}_{2}\right)$ ), where the subscript of the classes specifies their degree, $e_{4}$ is the image of the second Chern class of the natural representation of $S L_{2}(\mathbb{C})$, and all other classes are exterior classes.

This gives one more positive example for Quillen conjecture. Reader can find the details of the computation in ${ }^{6}$.

\section{CONCLUSION}

Over the last forty years, many results have been published about Quillen conjecture. Some give positive results, some introduce counterexamples in which the conjecture fails, and some make refinements. In ${ }^{11}$, Rahm and Wendt have stated that for large primes $\ell$, we can determine precisely the module structure above the virtual cohomological dimension . Therefore, the future work on this conjecture should focus on the cases of small primes. In order to refine the conjecture or find other counterexamples, there are two sources that we can look at:

- (i) The injectivity of the restriction map $H^{*}\left(G L_{n}\left(\mathbb{Z}\left[\frac{1}{2}\right]\right), \mathbb{F}_{2}\right) \rightarrow H^{*}\left(T_{n}\left(\mathbb{Z}\left[\frac{1}{2}\right]\right), \mathbb{F}_{2}\right)$ from $G L_{n}\left(\mathbb{Z}\left[\frac{1}{2}\right]\right)$ to the subgroup $T_{n}\left(\mathbb{Z}\left[\frac{1}{2}\right]\right)$ of diagonal matrices.

- (ii) In $^{12}$, Wendt found that the image of the Quillen homomorphism is not free in the cases that he is observing.

In order to examine a wide range of $S$-arithmetic groups, we may need to subdivide the spaces on that those groups act to get a rigid action. This problem may become a serious problem since those cell complexes can be very big and complicated. The first author and his collaborator have developed an algorithm named Rigid Facet Subdivision ${ }^{13}$ to overcome this problem.

\section{COMPETING INTERESTS}

The authors declare that they have no conflicts of interest.

\section{AUTHORS' CONTRIBUTIONS}

Nguyen Anh Thi have collected the information of Quillen's conjecture over

time and pointed out the two main keys to attack the conjecture by the work

of Henn and Wendt et al. Bui Anh Tuan provided an example which conrms

Quillen's conjecture.

\section{ACKNOWLEDGMENTS}

The authors were funded by Vietnam National University, Ho Chi Minh City (VNU- HCM) under grant number C2018-18-02. We would like to thank Alexander D. Rahm for having supported in the development of this project.

\section{REFERENCES}

1. Morris DW. Introduction to Arithmetic Groups. Deductive Press;. .

2. Quillen $D$. The spectrum of an equivariant cohomology ring. I, II Ann of Math. 1971;94(1):549-572. ibid. (2) 94 (1971), 573602.

3. Ostrowski A. Über einige Lsungen der Funktionalgleichung.. Acta Mathematica (2nd ed). 1916;41:271-284. Available from: 10.1007/BF02422947.ISSN0001-5962.

4. Mitchell SA. On the plus construction for $B G L(Z[1 / 2])$ at the prime 2. Math Z. 1992;209(2):205-222.

5. Henn HW. The cohomology of SL(3, Z[1/2]). K-Theory. 1999;16(4):299-359.

6. Bui AT, Rahm AD. Verification of the Quillen conjecture for Bianchi groups.; 2018. Submitted. 
7. Henn HW, Lannes J, Schwartz L. Localization of unstable Amodules and equivariant mod $\mathrm{p}$ cohomology. Math Ann. 1995;301:23-68.

8. Dwyer WG. Exotic cohomology for GLn(Z[1/2]). Proc Amer Math Soc. 1998;126(7):2159-2167.

9. Henn HW, Lannes J. Exotic classes in the mod 2 cohomology of $G \operatorname{Ln}(Z[1 / 2])$,. LEnseignement Math. 2008;54. special issue "Guido's book of conjectures," 107-108.

10. Anton MF. On a conjecture of Quillen at the prime 3. J Pure Appl Algebra. 1999;144(1):1-20.
11. Rahm AD, Wendt M. On Farrell-Tate cohomology of SL2 over S-integers. J Algebra. 2018;512:427-464.

12. Wendt M. On the cohomology of GL3 of elliptic curves and Quillen's conjecture, preprint, arXiv:1501.02613;

13. Bui AT, Rahm AD. Torsion Subcomplexes Subpackage", version 2.1, accepted sub-package in HAP (Homological Algebra Programming) in the computer algebra system GAP; 2018. Source code available at http://math.uni.lu/ rahm/subpackage- documentation/. 